\section{Questión}

Periodismo / Comunicación ISSN 1669-6581
- Av. $44 \mathrm{~N}^{\circ} 676,1^{\circ} \mathrm{piso}$

CP 1900 - La Plata - Argentina

@ www.perio.unlp.edu.ar/question

UNLP y la continuidad educativa a distancia como una opción pedagógica de consolidación del Derecho a la Educación Alejandro González - Carlos Milito

https://doi.org/10.24215/16696581e373

\title{
UNLP y la continuidad educativa a distancia como una opción pedagógica de consolidación del Derecho a la Educación
}

\section{UNLP and distance educational continuity as a pedagogical option to consolidate the Right to Education}

\begin{abstract}
Entrevista sonora a Alejandro González, Director General de Educación a Distancia y
Tecnologías de la Universidad Nacional de la Plata por Carlos Milito
\end{abstract}

\section{Resumen}

Las Universidades Nacional de La Plata decidió seguir dando clases en la emergencia de la pandemia, apelando a las posibilidades que brinda la educación mediada por tecnologías. La institución entiende que esa continuidad pedagógica es una forma de hacer efectivo el derecho a la educación, y de renovar el compromiso universitario para con la sociedad y los estudiantes. Alejandro González gestiona la anomalía desde la Dirección General de Educación a Distancia y Tecnologías de la Universidad Nacional de la Plata. En la entrevista da cuenta del proceso, del estado actual de situación. También destaca que sostener la actividad de enseñanza aprendizaje de manera virtual es una obligación política y pedagógica de la institución.

\section{Palabras Claves}

Educación a distancia - Continuidad educativa virtual - Pandemia - Universidad - Derecho a la Educación - Tecnologías

\section{Summary}

The National Universities of La Plata decided to continue teaching in the emergence of the pandemic, appealing to the possibilities offered by technology-mediated education. The institution 
understands that this pedagogical continuity is a way of making education effective and renewing university commitment to society and students. Alejandro González manages the anomaly from the General Directorate of Distance Education and Technologies of the National University of La Plata. In the interview he realizes the process, the current state of situation. It also stresses that sustaining the learning teaching activity in a virtual way is a political and pedagogical obligation of the institution.

\section{Keywords}

Distance education - Virtual educational continuity - Pandemic - University - Right to Education - Technologies

Alejandro González alejandro.gonzalez@presi.unlp.edu.ar Mg. en Tecnología Informática Aplicada en Educación, Especialista en Docencia Universitaria, Licenciado en informática y Analista en Computación. Actualmente es Director General de Educación a Distancia y Tecnologías de la Universidad Nacional de la Plata y responsable del SIED UNLP (Sistema institucional de educación a distancia de la UNLP) Investigador en Tecnologías Informáticas Aplicadas en Educación en el Instituto de Investigación en Informática III-LIDI de la Universidad Nacional de la Plata.

Coordinador en el diseño y ejecución de proyectos o programas de formación y actualización profesional y de integración de nuevas tecnologías en la educación. Asesor en el diseño de políticas, estrategias de incorporación o desarrollo de programas de uso de tecnologías para diversos ámbitos y sectores; Fue coordinador de RUEDA (Red de Universidades Argentinas de Educación a Distancia) por el período 2015-2017. Ex secretario Técnico de AULA-CAVILA: Asociación de Universidades Latinoamericanas - Campus Virtual Latinoamericano (2012-2015). 


\section{Entrevista}

Mag. Carlos Milito. https://orcid.org/0000-0002-5874-6951 cmilito@perio.unlp.edu.ar Magister en Comunicación y gestión de procesos comunicacionales FPyCS UNLP Profesor Titular del Taller de producción de contenidos y narrativas sonoras y radiales Director de la Especialización en Comunicación radiofónica - FPyCS UNLP Coordinador de 1894radioonline de Villa Elisa, Provincia de Buenos Aires Locutor comercial de Relatores. Transmisiones Deportivas por la app y AM530 Somos Radio

\section{Enlace}

https://ar.ivoox.com/es/51717541 